\title{
Interfacial charges and electric field distribution in organic hetero-layer light-emitting devices
}

\author{
Stefan Berleb and Wolfgang Brütting* \\ University of Bayreuth, Experimental Physics II, 95440 Bayreuth, Germany \\ Gernot Paasch \\ Institute for Solid State and Materials Research, 01171 Dresden, Germany
}

\begin{abstract}
The electric field distribution in organic hetero-layer light-emitting devices based on $\mathrm{N}, \mathrm{N}^{\prime}$-diphenyl-N,N'-bis(1-naphtyl)-1,1'-biphenyl-4,4'-diamine (NPB) and 8-tris-hydroxyquinoline aluminium $\left(\mathrm{Alq}_{3}\right)$ has been investigated under different bias conditions using capacitance-voltage measurements. Although this method yields primarily information on the differential capacitance, the data give clear evidence for the presence of negative interfacial charges with a density of $6.8 \times 10^{11} \mathrm{e} / \mathrm{cm}^{2}$ at the $\mathrm{NPB} / \mathrm{Alq}_{3}$ interface at large reverse bias. This leads to a jump of the electric field at the interface and a non-uniform field distribution in the hetero-layer device.
\end{abstract}

A major breakthrough in the development of organic electroluminescent devices for display applications was the demonstration of high efficiency and brightness at moderate voltages by means of an organic multi-layer device $[1,2]$. The underlying idea was to separately optimize injection and transport of holes and electrons and their radiative recombination in different organic layers. A common starting point for device optimization is the considera-

*Electronic mail: Wolfgang.Bruetting@uni-bayreuth.de 
tion of molecular energy levels, which are relevant for the magnitude of the energy barriers at the injecting contacts and at the organic-organic interfaces. Although the knowledge of these energy levels is an important guide for the choice of an appropriate combination of organic materials and electrodes, it is not enough for describing the energetic situation in an operating device. The reason is that the accumulation of charge carriers as interface charges or dipoles can significantly modify the electrical potential inside the device. From current-voltage characteristics alone only very indirect conclusions about the field distribution can be drawn, necessitating detailed numerical simulations, a procedure which is not unambiguous, because many of the material parameters are not known with the necessary accuracy $[3-6]$.

We have based our present investigations on the frequently used two-layer heterostructure ITO/NPB/Alq $q_{3} / \mathrm{Ca}$, consisting of a triaryl-amine derivative (N,N'-diphenyl-N,N'-bis(1naphtyl)-1,1'-biphenyl-4,4' -diamine, NPB) as hole-transport-layer and an aluminium metal chelate complex (8-tris-hydroxyquinoline aluminium, $\mathrm{Alq}_{3}$ ) as electron transport and emissive material sandwiched between an indium-tin oxide (ITO) anode and a Ca cathode. The chemical formulae and energy levels of these materials are shown in Figure 1. For this type of hetero-layer devices it has been concluded already from the analysis of current-voltage $(I-V)$ characteristics by Matsumura et al. [7] and from electroabsorption spectroscopy by Rohlfing et al. [8] that under forward bias the voltage drop at the $\mathrm{Alq}_{3}$ layer is much larger than at the NPB layer. This is not unexpected since an accumulation of injected holes at the $\mathrm{NPB} / \mathrm{Alq}_{3}$ interface will occur due to the energy barrier for holes and also since the hole mobility in NPB is much larger than in $\mathrm{Alq}_{3}$. The present work is focused on the device behaviour under reverse bias using low-frequency capacitance-voltage $(C-V)$ measurements. It will be shown that the measurements give clear evidence for the presence of interfacial charges in the voltage range where no carrier injection occurs. Although $C-V$ measurements are only a small part of a full impedance spectroscopic analysis including the frequency, bias and temperature dependence of the impedance (see e.g. Ref. [9]), they already reveal unique information about interfacial charges in this system so far not reported 
in the literature.

The materials were obtained from commercial suppliers: NPB from Syntec and Alq from Aldrich or Dojindo. Although the purity of these materials has significant influence on the magnitude of the current under forward bias operation, we found that the phenomenon reported in the present paper is not affected by this issue. ITO-coated glass substrates from Merck were used as anode. The ITO substrates were cleaned in an ultrasonic bath with detergent, acetone and deionized water, followed by drying in isopropanol vapour. Immediately before loading into the evaporation chamber the ITO substrates were oxygen plasma-treated to increase the work function, which significantly improves hole injection into NPB. The organic materials were deposited in a vacuum deposition chamber at a base pressure of $10^{-6} \mathrm{mbar}$ and with deposition rates of $1 \AA / \mathrm{s}$. Calcium electrodes were vapourdeposited under the same conditions with a shadow mask; the resulting device area was $8.2 \mathrm{~mm}^{2}$. Devices with different film thickness were fabricated in a single vacuum cycle, also the Ca electrodes were deposited simultaneously for the different devices. The deposition chamber was coupled to a glovebox system where the devices were stored and measured under dry nitrogen atmosphere. Impedance measurements were carried out with an impedance analyzer (Hewlett Packard 4194A) and a frequency response analyzer (Solartron Instruments SI1260) combined with a broadband dielectric converter (Novocontrol). The used oscillator level (a.c. amplitude) was $100 \mathrm{mV}$ r.m.s. To determine the influence of the different layers we systematically varied the thickness of the NPB and the $\mathrm{Alq}_{3}$ layers between 0 and 240nm. All measurements reported here were carried out at room temperature.

Typical data of the complex impedance $\hat{Z}=Z^{\prime}+i Z^{\prime \prime}=|\hat{Z}| \cdot e^{i \varphi}$ are shown in Figure 2 for an $\mathrm{ITO} / \mathrm{NPB}(60 \mathrm{~nm}) / \mathrm{Alq}_{3}(60 \mathrm{~nm}) / \mathrm{Ca}$ device in the representation of the modulus of the impedance $|\hat{Z}|$ and the phase $\varphi$. For large reverse bias the device behaves essentially like an insulating dielectric with a purely capacitive response $(\varphi=-\pi / 2)$. Above a turn-on voltage in the range 2 to $2.5 \mathrm{~V}$, the phase $\varphi$ begins to deviate from $-\pi / 2$ and changes to 0 , indicating conducting behaviour, and the modulus of the impedance $|\hat{Z}|$ also decreases rapidly with increasing voltage due to carrier injection. This turn-on voltage can approximately be 
identified with the built-in voltage $V_{\mathrm{bi}}$ corresponding to the contact potential difference of the ITO and Ca electrodes (ignoring interface dipoles at the metal electrodes), which has to be overcome before a non-negligible drift current can flow [10,11]. Below the built-in voltage an additional feature appears at about $-0.5 \mathrm{~V}$. A weak bump occurs in the phase and $|\hat{Z}|$ decreases by a factor of 5 to a new plateau value. This feature has no equivalent in the $I-V$ characteristics (see e.g. Ref. [6,12]). The current below $V_{\mathrm{bi}}$ is usually very low $\left(10^{-8} \mathrm{~A} / \mathrm{cm}^{2}\right.$ or less) and varies almost linearly with the applied voltage between $V_{\mathrm{bi}}$ and large negative values down to $-10 \mathrm{~V}$ without showing any additional structure.

We have investigated this unexpected behaviour of the complex impedance below $V_{\mathrm{bi}}$ in detail by systematically varying the thickness of the NPB and the Alq layer independently. For the sake of simplicity we will only discuss the differential capacitance $C$ at a fixed frequency $f$, which is obtained from the complex impedance as [13]:

$$
C=(1 / 2 \pi f) \cdot\left(-Z^{\prime \prime}\right) /\left(Z^{\prime 2}+Z^{\prime \prime 2}\right)
$$

Results are shown here for $f=100 \mathrm{~Hz}$, but they are independent of the measurement frequency up to about $10 \mathrm{kHz}$ [14]. A full analysis of the frequency and temperature dependence is under way and will be published elsewhere. Figure 3 shows two groups of data for devices with 60 and 120nm Alq $\mathrm{n}_{3}$ layer thickness combined with NPB thicknesses of 0,30 and $60 \mathrm{~nm}$. In both data sets two different voltage regimes separated by a transition voltage $V_{0}$ (indicated by arrows) can be identified. For voltages well below $V_{0}$ the measured value of the capacitance depends on the overall thickness of both organic layers and is given by $C^{-1}=C_{\mathrm{NPB}}^{-1}+C_{\mathrm{Alq}_{3}}^{-1}$ for the two dielectrica in series. For equal dielectric constants the capacitance can be also expressed as $C=\varepsilon_{\mathrm{r}} \varepsilon_{0} A / d$ where $A$ is the device area and $d$ the total organic layer thickness. Here we have used a dielectric constant $\varepsilon_{\mathrm{r}}=3.5$ for both $\mathrm{NPB}$ and $\mathrm{Alq}_{3}$ which has been determined from the capacitance of single layer devices. The equality of the $\varepsilon_{\mathrm{r}}$ values can be directly seen in Figure $\mathbf{3}$ by the fact that the capacitance of the device with $60 \mathrm{~nm} \mathrm{NPB}$ and $60 \mathrm{~nm} \mathrm{Alq}_{3}$ is below $-3 \mathrm{~V}$ almost the same as with $120 \mathrm{~nm} \mathrm{Alq} 3$ alone. When the voltage is increased above a critical value (of about $-3 \mathrm{~V}$ 
for $d_{\mathrm{Alq}_{3}}=120 \mathrm{~nm}$ and of about $-1 \mathrm{~V}$ for $d_{\mathrm{Alq}_{3}}=60 \mathrm{~nm}$ ), however, the capacitance increases and is already $1 \mathrm{~V}$ above this voltage approximately equal to the capacitance of the $\mathrm{Alq}_{3}$ layer alone: $C=C_{\mathrm{Alq}_{3}}=\varepsilon_{\mathrm{r}} \varepsilon_{0} A / d_{\mathrm{Alq}_{3}}$. It should be emphasized that the transition does not occur at the built-in voltage, but at a transition voltage $V_{0}$ which is lower than $V_{\mathrm{bi}}$ and even negative. The steep increase of the capacitance around $V_{0}$ occurs over a range of about $1 \mathrm{~V}$, which is larger than the oscillator amplitude and not influenced by it, as long as the a.c. amplitude stays below about $0.5 \mathrm{~V}$.

It is evident from Figure 3 that the transition voltage $V_{0}$ is independent of the NPB layer thickness. We also found the value of $V_{0}$ not to be frequency dependent for measurement frequencies below $10 \mathrm{kHz}$ [14]. However, as already seen in Figure $\mathbf{3}, V_{0}$ is significantly different for $\mathrm{Alq}_{3}$ thicknesses of 60 and 120nm. Figure 4 shows in more detail the dependence of $V_{0}$ on the $\mathrm{Alq}_{3}$ thickness. Here the thickness of the NPB layer was kept constant at $60 \mathrm{~nm}$ and the $\mathrm{Alq}_{3}$ thickness varied from 10 to $240 \mathrm{~nm}$. All curves show the above described increase from a lower capacitance value at large reverse bias corresponding to the total organic layer thickness to a higher value given by the $\mathrm{Alq}_{3}$ thickness. The voltage, however, where the transition occurs, critically depends on the thickness of the $\mathrm{Alq}_{3}$ layer. Obviously, the transition voltage is close to the built-in voltage for the thinnest $\mathrm{Alq}_{3}$ layer and shifts towards zero and negative voltage with increasing $\mathrm{Alq}_{3}$ thickness. The inset of Figure 4 shows a plot of the transition voltage $V_{0}$ (taken as the inflection point of the $C-V$ curves) vs. the $\mathrm{Alq}_{3}$ thickness. Within the error limits given by the width of the transition and the thickness measurement the transition voltage depends linearly on the $\mathrm{Alq}_{3}$ thickness. The fitted straight line intersects the voltage axis at $(2.2 \pm 0.15) \mathrm{V}$, which is very close to the built-in voltage of 1.9 to $2.2 \mathrm{~V}$, estimated from $I-V$ characteristics [12], and its slope is $(-0.35 \pm 0.03) \mathrm{MV} / \mathrm{cm}$. We note that the value of the slope is not affected by the definition of the transition voltage $V_{0}$. If the onset of capacitance increase were taken instead of the inflection point, only a lower value of the intersection with the voltage axis of about $1.9 \mathrm{~V}$ would have been obtained.

The behaviour of the device capacitance described above reveals four characteristic fea- 
tures: (i) For large negative bias $\left(V \ll V_{0}\right)$ the measured differential capacitance is equal to the geometric capacitance of both layers: $C^{-1}=C_{\mathrm{NPB}}^{-1}+C_{\mathrm{Alq}_{3}}^{-1}$. (ii) In the voltage range $V_{0}<V<V_{\mathrm{bi}}$ the capacitance increases and for $V=V_{\mathrm{bi}}$ only the capacitance of the Alq layer is obtained: $C=C_{\mathrm{Alq}_{3}}$. (iii) Above $V_{\mathrm{bi}}$ the device behaviour changes from capacitive to conductive (the phase turns from $-\pi / 2$ to 0 ). (iv) The critical voltage $V_{0}$ is determined by the $\mathrm{Alq}_{3}$ thickness and a critical field $E_{0}=-0.35 \mathrm{MV} / \mathrm{cm}$, where the critical field is defined by the relation: $V_{0}-V_{\mathrm{bi}}=E_{0} d_{\mathrm{Alq}_{3}}$.

For the interpretation to be given another experimental observation is important to mention: The $I-V$ characteristics of ITO/NPB/Ca (and also ITO/Alq $/ \mathrm{Ca}$ ) devices show a strongly rectifying behaviour. This means that for voltages below $V_{\mathrm{bi}}$ the current is by orders of magnitude lower than for $V>V_{\mathrm{bi}}[12]$. Thus, the differential resistance $R=(\mathrm{d} I / \mathrm{d} V)^{-1}$ is also many orders of magnitude larger for reverse bias than for $V>V_{\mathrm{bi}}$.

A consistent description of these observations is obtained by supposing at first the existence of a negative charge $Q_{\text {if }}=Q_{0}<0$ at the NPB/Alq $\mathrm{Al}_{3}$ interface for large negative bias $V \ll V_{0}$. This charge is compensated by positive charges $-Q_{0} d_{\mathrm{NPB}} / d$ on the Ca cathode and $-Q_{0} d_{\mathrm{Alq}_{3}} / d$ on the ITO anode $\left(d=d_{\mathrm{Alq}_{3}}+d_{\mathrm{NPB}}\right)$. Further, for a given applied bias there are the charges $Q$ (resp. $-Q$ ) sitting on the anode (resp. cathode). For the geometry as chosen in Figure 5 the electric field is given in the two layers by [15]

$$
\begin{aligned}
& E_{\mathrm{NPB}}=\frac{Q-Q_{0} d_{\mathrm{Alq}_{3}} / d}{\varepsilon_{\mathrm{r}} \varepsilon_{0} A}, \\
& E_{\mathrm{Alq}_{3}}=\frac{Q+Q_{0} d_{\mathrm{NPB}} / d}{\varepsilon_{\mathrm{r}} \varepsilon_{0} A}
\end{aligned}
$$

with a jump of the electric field at the $\mathrm{NPB} / \mathrm{Alq}_{3}$ interface

$$
E_{\mathrm{Alq}_{3}}-E_{\mathrm{NPB}}=E_{0}=\frac{Q_{0}}{\varepsilon_{\mathrm{r}} \varepsilon_{0} A}=\frac{\sigma_{0}}{\varepsilon_{\mathrm{r}} \varepsilon_{0}}
$$

The anode voltage is independently of the interfacial charge $Q_{0}$ given by

$$
V-V_{\mathrm{bi}}=\frac{d_{\mathrm{NPB}}}{\varepsilon_{\mathrm{r}} \varepsilon_{0} A} Q+\frac{d_{\mathrm{Alq}_{3}}}{\varepsilon_{\mathrm{r}} \varepsilon_{0} A} Q
$$


and the capacitance follows from

$$
C^{-1}=\frac{\mathrm{d} V}{\mathrm{~d} Q}=\frac{d_{\mathrm{NPB}}}{\varepsilon_{\mathrm{r}} \varepsilon_{0} A}+\frac{d_{\mathrm{Alq}_{3}}}{\varepsilon_{\mathrm{r}} \varepsilon_{0} A}=C_{\mathrm{NPB}}^{-1}+C_{\mathrm{Alq}_{3}}^{-1}
$$

as the series connection of the capacitances of the two layers. For sufficiently large negative bias the electric fields in both layers (Eqs. (2) and (3)) are negative and the absolute value of the field in the $\mathrm{Alq}_{3}$ layer is larger than in the NPB layer $\left(Q<0\right.$ and $\left.Q_{0}<0\right)$. The corresponding electric potential is depicted in Figure 5a (as the potential energy for an electron). In this voltage range both the NPB and Alq layer are biased in reverse direction and have therefore such a high resistance that at the measurement frequency $\omega=2 \pi f$ the condition $\omega \tau \gg 1$ with $\tau=R C$ holds for both layers and the two capacitors are measured in series (see e.g. Ref. [16] for a detailed discussion of the impedance of a double $R C$ network).

When the bias increases one reaches the flat band case in the NPB layer with zero electric field there and hence an induced charge $Q=Q_{0} d_{\mathrm{Alq}_{3}} / d(\mathrm{Eq} .(2))$. Consequently there is only the electric field in the $\mathrm{Alq}_{3}$ layer with a magnitude equal to the jump caused by the interfacial charge density $\sigma_{0}$ (Eqs. (3) and (4)): $E_{\mathrm{Alq}_{3}}=E_{0}$. The anode voltage is for this case just

$$
V_{0}-V_{\mathrm{bi}}=\frac{d_{\mathrm{Alq}_{3}}}{\varepsilon_{\mathrm{r}} \varepsilon_{0} A} Q_{0}=E_{0} d_{\mathrm{Alq}_{3}}
$$

In this way the transition voltage $V_{0}$ is connected with the critical field as deduced from the experiment. From Eq. (4) for the above obtained value of the critical field this yields an interfacial charge density of $\sigma_{0}=-1.1 \times 10^{-7} \mathrm{As} / \mathrm{cm}^{2}=-6.8 \times 10^{11} \mathrm{e} / \mathrm{cm}^{2}$ (e being the elementary charge). Since for this applied voltage the field drops completely over the $\mathrm{Alq}_{3}$ layer and the NPB layer has reached the flat band condition (see Figure $5 \mathbf{b}$ ) the resistance of the NPB layer drastically drops so that now $\omega \tau_{\mathrm{NPB}} \ll 1$ whereas in $\mathrm{Alq}_{3}$ still $\omega \tau_{\mathrm{Alq}_{3}} \gg 1$ holds. Under these conditions only the capacitance of the layer with the higher resistance is measured, i.e. that of the $\mathrm{Alq}_{3}$ layer (cf. again Ref. [16]).

For $V_{0}<V<V_{\mathrm{bi}}$ it would in principle be possible that the total interfacial charge $Q_{\text {if }}$ remains equal to $Q_{0}$. However, if this were the case, the electric field would have different 
sign in both organic layers and the electric potential would show a maximum at the interface. Furthermore, at $V=V_{\mathrm{bi}}$ the flat band condition would not be achieved, and the Alqu layer would be still reverse biased. This is in clear contradiction to the strong increase of the current in the $I-V$ characteristics immediately above $V_{\mathrm{bi}} \approx 2 \mathrm{~V}[6,12]$. Therefore, it is reasonable to assume that for $V>V_{0}$, as soon as the flat band condition for NPB is achieved, holes can be injected at the anode and pass through the NPB layer to reach the interface. There they will partly compensate the fixed negative interfacial charge $Q_{0}$ and the total interfacial charge $\left|Q_{\text {if }}\right|$ becomes less than $\left|Q_{0}\right|$ such that the NPB layer stays in the flat band condition (see Figure 5c). Formally speaking, one has to replace $Q_{0}$ in Eqs. (2) and (3) by $Q_{\text {if }}$, which varies with the applied bias in a way that the field in the NPB layer (Eq. (2)) vanishes for $V_{0}<V<V_{\mathrm{bi}}$. Then the total differential capacitance is in this voltage range given by the capacitance of the $\mathrm{Alq}_{3}$ layer

$$
C^{-1}=\frac{\mathrm{d} V}{\mathrm{~d} Q}=\frac{d_{\mathrm{Alq}_{3}}}{\varepsilon_{\mathrm{r}} \varepsilon_{0} A}=C_{\mathrm{Alq}_{3}}^{-1}
$$

since one has still $\omega \tau_{\mathrm{NPB}} \ll 1$ and $\omega \tau_{\mathrm{Alq}_{3}} \gg 1$. We note that the scenario described above implies that the ITO anode has good hole injecting properties. Indeed, we do not observe this effect if the oxygen plasma-treatment is omitted, which is known to enhance the ITO work function considerably [17].

With further increasing voltage the total interfacial charge $\left|Q_{\text {if }}\right|$ will be reduced gradually until at $V_{\mathrm{bi}}$ the fixed charge $Q_{0}$ is fully neutralized and both the NPB and $\mathrm{Alq}_{3}$ layer have flat bands (see Figure 5d). Above $V_{\text {bi }}$ the device is forward biased and carrier double injection occurs. Due to the strong difference in charge carrier mobility between NPB and $\mathrm{Alq}_{3}\left(\mu_{\mathrm{h}, \mathrm{NPB}} \gg \mu_{\mathrm{e}, \mathrm{Alq}_{3}} \gg \mu_{\mathrm{h}, \mathrm{Alq}_{3}}\right)[18-20]$ the voltage drop at the Alq $\mathrm{q}_{3}$ layer will be still much higher than at the NPB layer (see for example Ref. [7,8]), but now the origin is an accumulation of injected positive carriers at the interface (see Figure 5e).

Thus the assumption of a negative interfacial charge at the $\mathrm{NPB} / \mathrm{Alq}_{3}$ interface which is constant for $V \ll V_{0}$ and becomes gradually compensated for $V_{0}<V<V_{\text {bi }}$ is inevitable to explain the observed capacitance-voltage measurements. At present, there is little direct 
information about the microscopic origin of the interfacial charge $Q_{0}$. It is a property of the $\mathrm{NPB} / \mathrm{Alq}_{3}$ interface, which is not dependent on external parameters like layer thickness, electrodes or material purification. Even if an additional $\mathrm{Cu}$-phthalocyanine layer is inserted between ITO and NPB the transition voltage for a given $\mathrm{Alq}_{3}$ thickness is not changed [21]. However, preliminary experiments have shown that if an oxadiazole is used instead of $\mathrm{Alq}_{3}$ the magnitude of $Q_{0}$ increases. A possible explanation could be a partial charge transfer between the hole and electron conducting material in the hetero-layer device. A similar effect was observed by photoelectron spectroscopy by Rajagopal et al. [22], who interpreted the shift of vacuum level positions at the $\mathrm{NPB} / \mathrm{Alq}_{3}$ interface by a partial electron transfer from $\mathrm{Alq}_{3}$ to NPB. However, such a charge transfer would lead to a dipole layer without net charge [23]. Only if one type of charges in the dipole layer is able to move away from the interface or can be compensated by charges of opposite sign in the respective organic layer, a net charge will remain. Another possibility are deep acceptor-like traps at the interface between both materials which have been found by numerical simulations to reproduce well

the observed bias and thickness dependence of the capacitance [24]. Therefore, further investigations with different experimental techniques will be necessary to get more insight into the microscopic nature of interfaces in organic hetero-layer LEDs.

\section{Acknowledgment}

We thank T. Schoberth and A. Mückl for experimental assistance and W. Rieß (IBM Zurich) for helpful discussions. Financial support from the Deutsche Forschungsgemeinschaft is gratefully acknowledged. 


\section{REFERENCES}

[1] C.W. Tang, S.A. VanSlyke, Appl. Phys. Lett. 51, 913 (1987).

[2] C.W. Tang, S.A. VanSlyke, C.H. Chen, J. Appl. Phys. 65, 3610 (1989).

[3] P.E. Burrows, Z. Shen, V. Bulovic, D.M. McCarty, S.R. Forrest, J.A. Cronin, M.E. Thomson, J. Appl. Phys. 79, 7991 (1996).

[4] J. Shen, J. Yang, J. Appl. Phys. 83, 7706 (1998).

[5] A. Ioannidis, E. Forsythe, Y. Gao, M.W. Wu, E.M. Conwell, Appl. Phys. Lett. 72, 3038 (1998).

[6] J. Staudigel, M. Stößel, F. Steuber, J. Simmerer, J. Appl. Phys. 86, 3895 (1999).

[7] M. Matsumura, A. Ito, Y. Miyamae, Appl. Phys. Lett. 75, 1042 (1999).

[8] F. Rohlfing, T. Yamada, T. Tsutsui, J. Appl. Phys. 86, 4978 (1999).

[9] J. Scherbel, P.H. Nguyen, G. Paasch, W. Brütting, M. Schwoerer, J. Appl. Phys. 83, 5045 (1998).

[10] J.C. Scott, S. Karg, S.A. Carter, J. Appl. Phys. 82, 1454 (1997).

[11] P.H. Nguyen, S. Scheinert, G. Paasch, S. Berleb, W. Brütting, to be published.

[12] S. Berleb, A. G. Mückl, W. Brütting, M. Schwoerer, Synth. Met. 111-112, 341 (2000).

[13] In an equivalent parallel circuit consisting of a resistor $R$ and a capacitor $C$ the complex admittance $\hat{Y}=\hat{Z}^{-1}$ is given by $\hat{Y}=R^{-1}+i \omega C$. Using $\hat{Z}=Z^{\prime}+i Z^{\prime \prime}$ then leads to equation (1).

[14] S. Berleb, W. Brütting, G. Paasch, Proc. E-MRS 2000, Synth. Met. (in press).

[15] The electric field in the organic layers is derived from the one-dimensional Poisson equation $\mathrm{d} E / \mathrm{d} x=\sigma / \varepsilon_{r} \varepsilon_{0}$ considering the charge densities $\sigma_{i}=Q_{i} / A$ at the metal-organic and organic-organic interfaces together with the boundary condition 
$\int_{0}^{d} E(x) \mathrm{d} x=V-V_{\mathrm{bi}}$ where the ITO anode is at $x=0$ and the Ca cathode at $x=d$ and equal dielectric constants $\varepsilon_{r}$ are taken.

[16] D.M. Taylor, H.L. Gomes, J. Phys. D: Appl. Phys. 28, 2554 (1995).

[17] K. Sugiyama, H. Ishii, Y. Ouchi, K. Seki, J. Appl. Phys. 87, 295 (2000).

[18] T. Tsutsui, H. Tokuhisa, M. Era, Polymer Photonic Devices, SPIE 3281, 230 (1998).

[19] S. Naka, H. Okada, H. Onnagawa, J. Kido, T. Tsutsui, Jpn. J. Appl. Phys. 38, L-1252 (1999).

[20] A.G. Mückl, S. Berleb, W. Bütting, M. Schwoerer, Synth. Met. 111-112, 91 (2000).

[21] W. Brütting, H. Riel, T. Beierlein, W. Riess, submitted to J. Appl. Phys. (2000).

[22] A. Rajagopal, C. I. Wu, A. Kahn, J. Appl. Phys. 83, 2649 (1998).

[23] H. Ishii, K. Sugiyama, E. Ito, K. Seki, Adv. Mat. 11, 605 (1999).

[24] S. Scheinert, G. Paasch, S. Berleb, W. Brütting, unpublished 
FIGURES

FIG. 1. Schematic configuration of the investigated hetero-layer devices together with chemical formulae and energy levels of the materials. All values are given in $\mathrm{eV}$.

FIG. 2. Modulus and phase of the complex impedance of an ITO/NPB $(60 \mathrm{~nm}) / \mathrm{Alq}_{3}(60 \mathrm{~nm}) / \mathrm{Ca}$ device as a function of the applied bias, measured at a frequency of $100 \mathrm{~Hz}$.

FIG. 3. Bias dependent device capacitance of a series of ITO/NPB/Alq $3 / \mathrm{Ca}$ devices with different NPB and Alq $\mathrm{Alayer}_{3}$ thicknesses.

FIG. 4. Bias dependent device capacitance of a series of ITO/NPB $/ \mathrm{Alq}_{3} / \mathrm{Ca}$ devices with a 60nm thick NPB layer and different $\mathrm{Alq}_{3}$ layer thicknesses. The inset shows the dependence of the transition voltage on the $\mathrm{Alq}_{3}$ layer thickness.

FIG. 5. Spatial dependence of the potential (energy) inside an ITO/NPB/Alq $/$ /Ca device under different bias conditions for equal thickness and dielectric constant of the two organic layers: (a) For large reverse bias the interfacial charge $Q_{\mathrm{if}}=Q_{0}<0$ creates a jump of the electrical field, which is equivalent to the change of slope of the potential at the interface. The dashed line indicates the situation without interfacial charge. (b) At $V=V_{0}$ the flat band condition is reached in NPB. (c) For $V_{0}<V<V_{\mathrm{bi}}$ NPB stays in the flat band condition, the amount of negative charge at the interface becomes smaller and concomitantly the jump of the electric field is reduced. (d) At $V=V_{\mathrm{bi}}$ the flat band condition in both layers is reached and the interfacial charges are fully neutralized. (e) For $V>V_{\mathrm{bi}}$ injected positive carriers accumulate at the interface to generate an opposite jump of the electric field. 
Figure 1

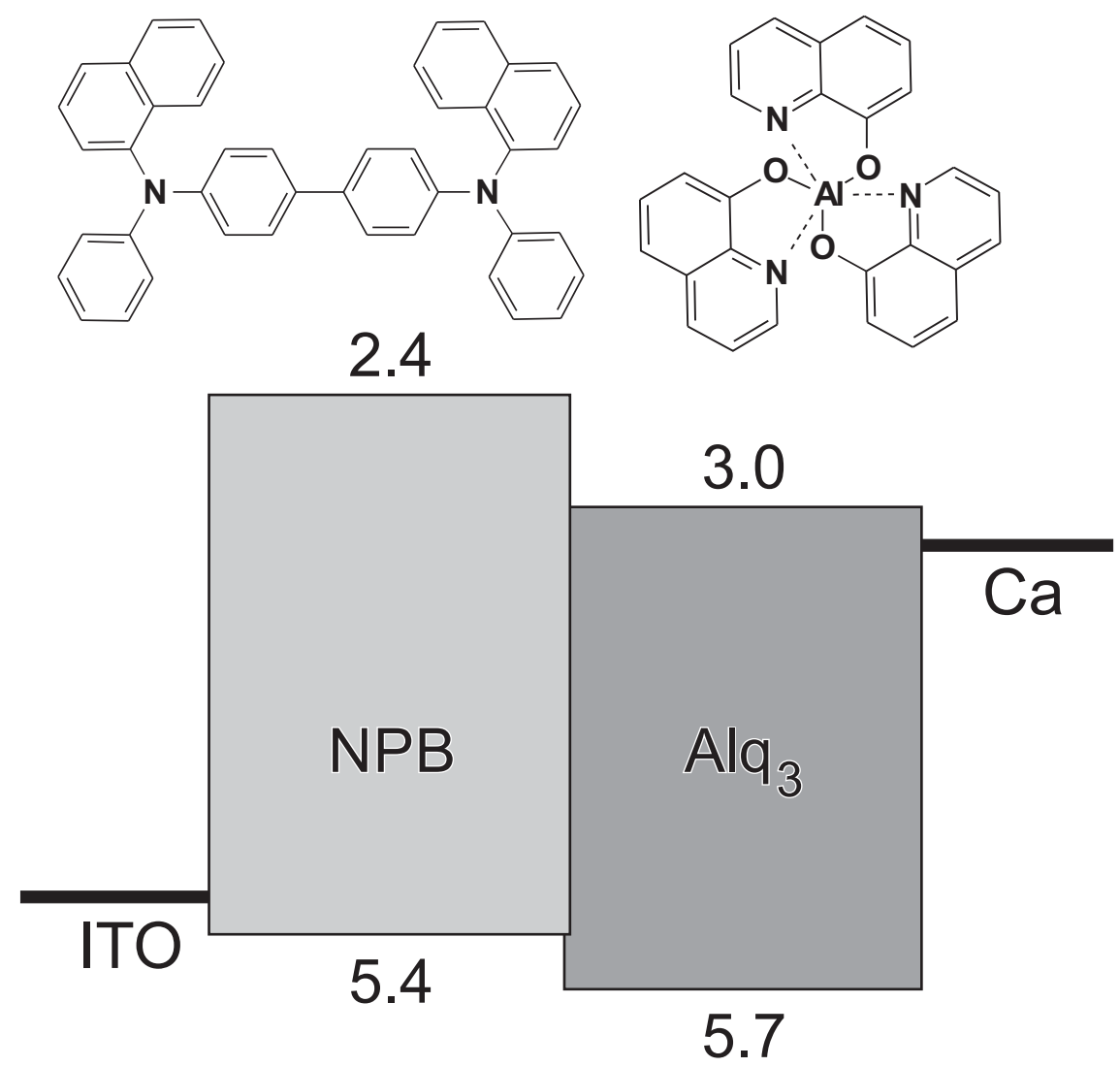


Figure 2

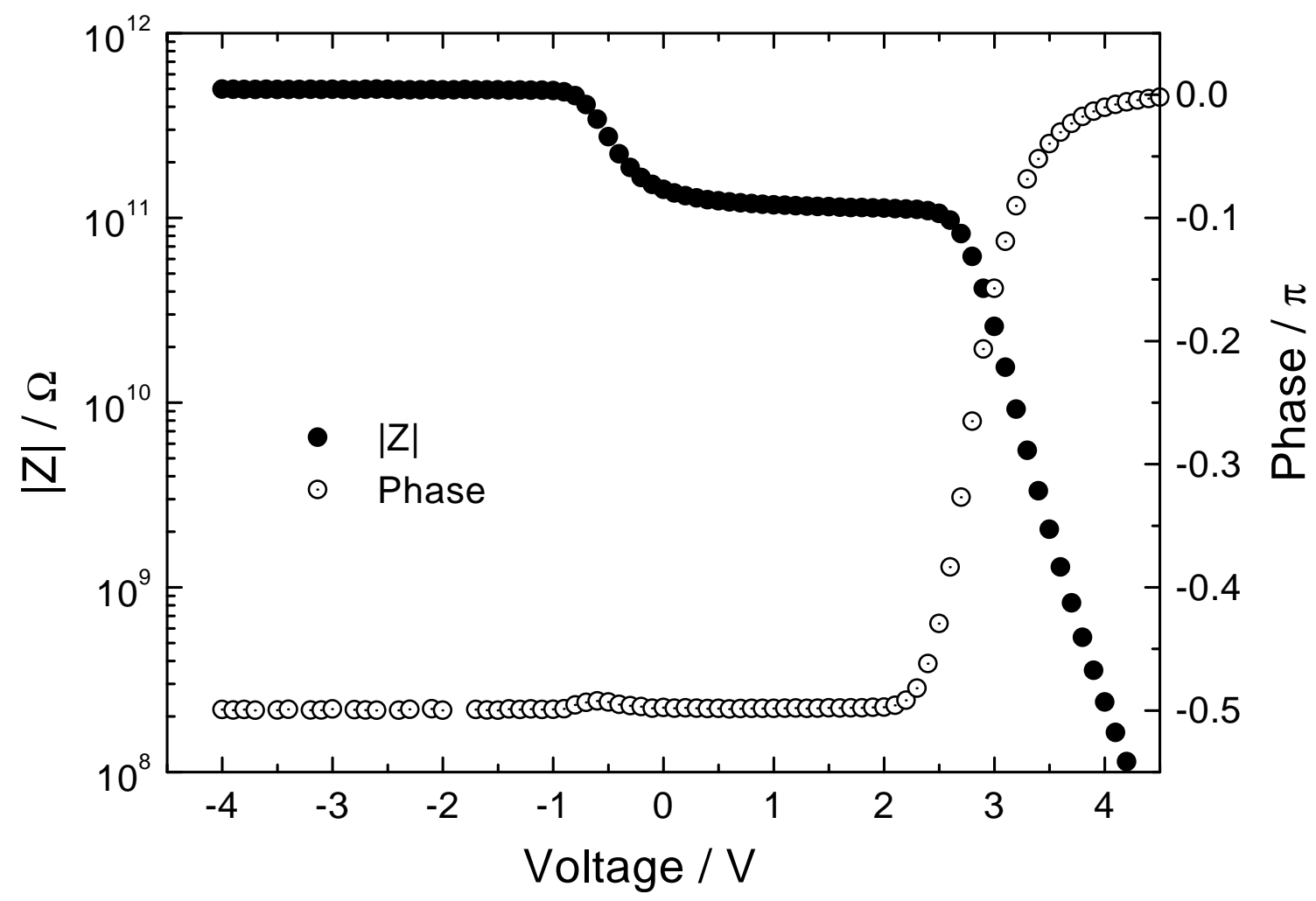


Figure 3

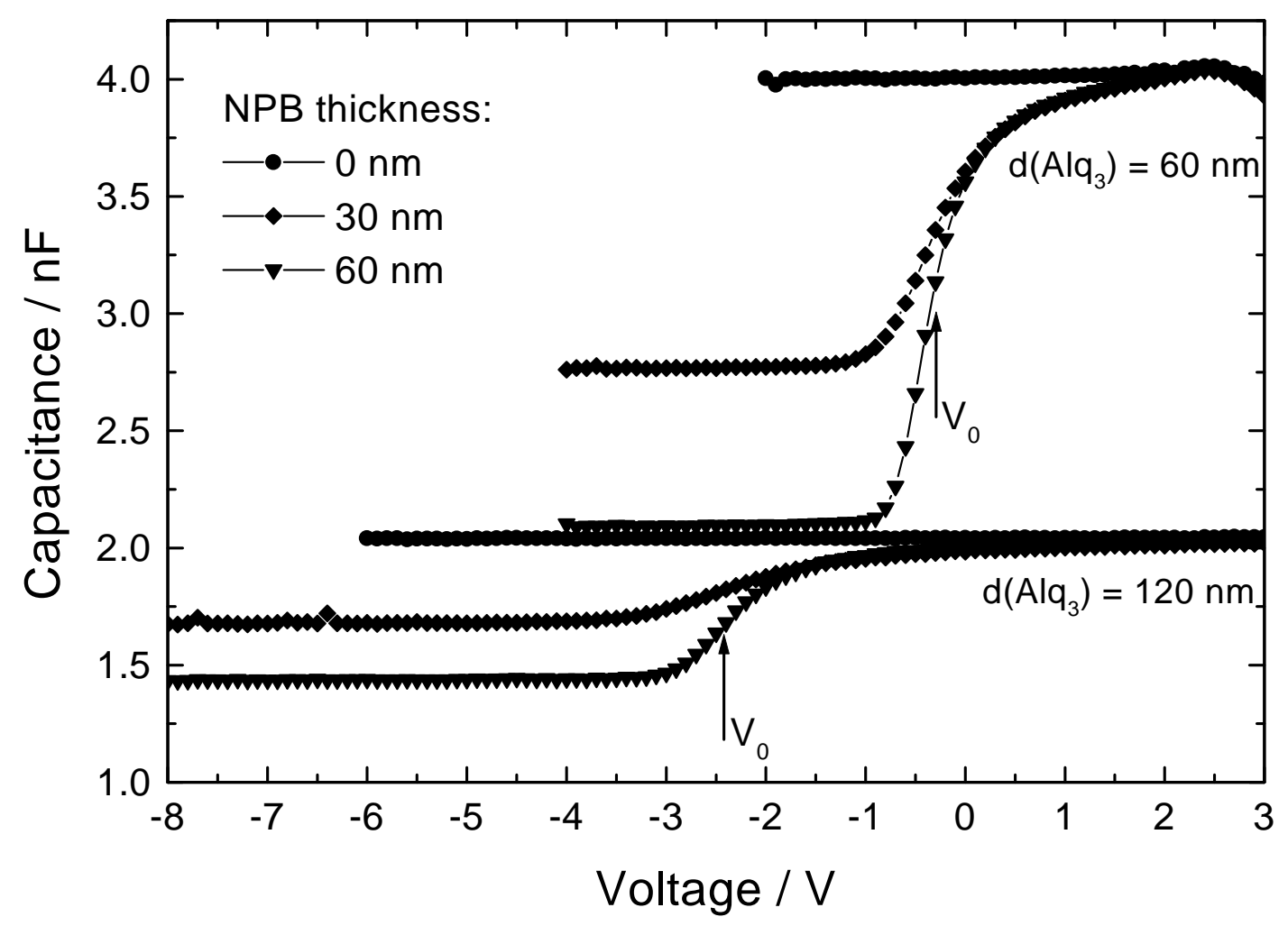


Figure 4

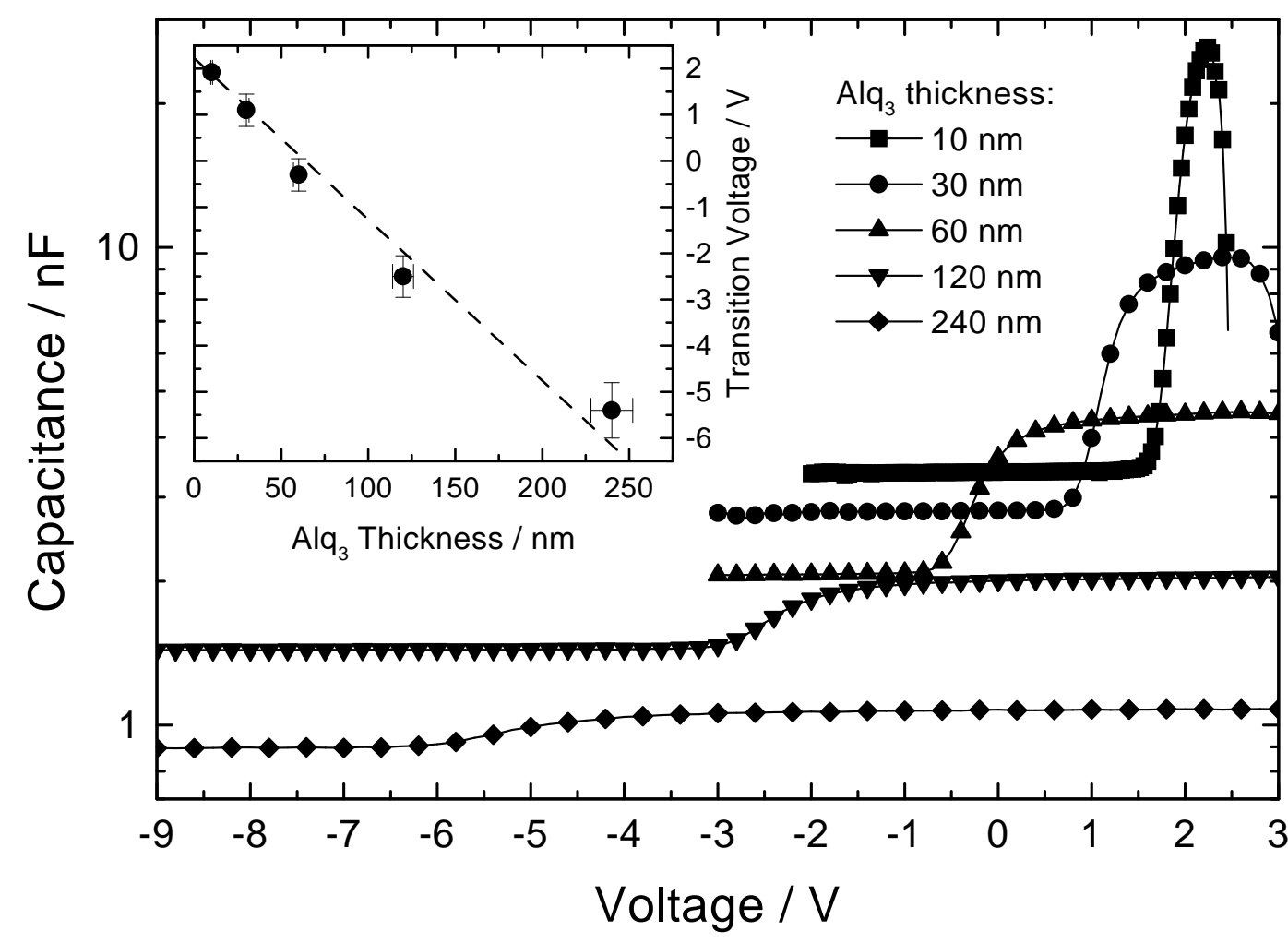


Figure 5

(a)

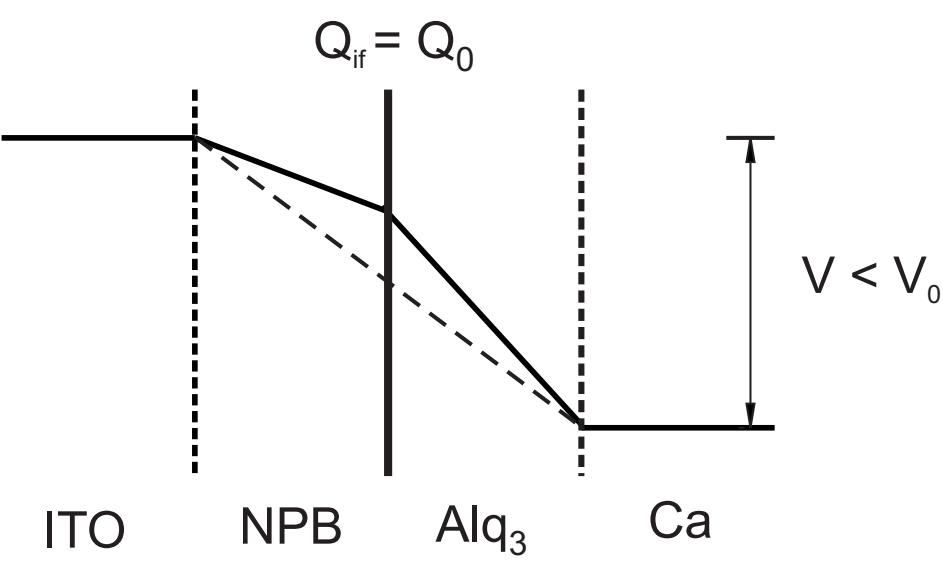

$Q_{\text {if }}=Q_{0}$

(b)

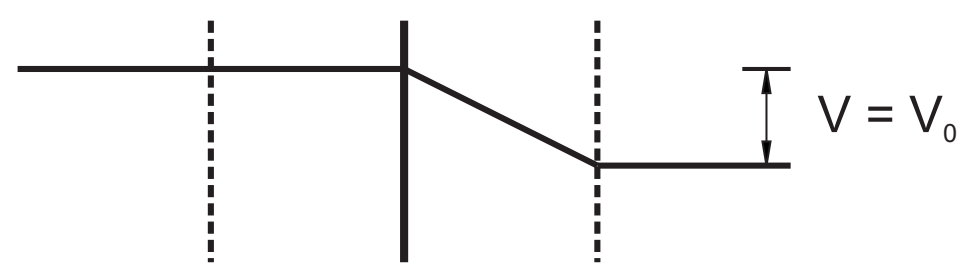

(c)

$$
Q_{0}<Q_{\text {if }}<0
$$

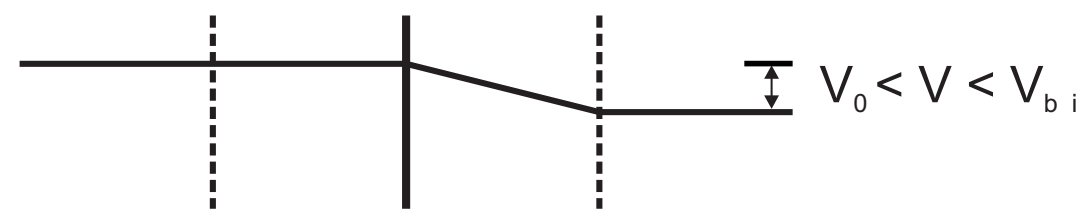

(d)

$$
Q_{\text {if }}=0
$$

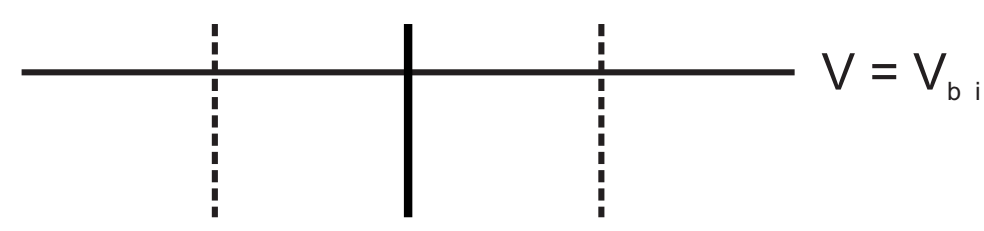

(e)

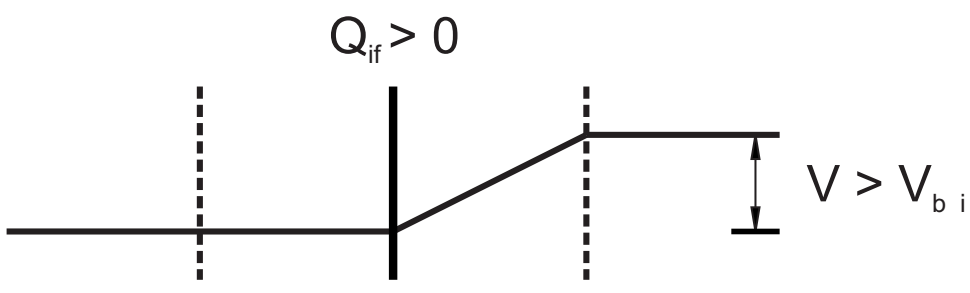

